UDC 621.891

\title{
INFLUENCE OF BORINATION, BORON CEMENTATION OF STEEL 45 AND VISCOSITY OF A LUBRICANT ON THE PARAMETERS OF ADHESION BOND IN THE STEEL 45 - 40X SYSTEM
}

\author{
Kubich V.I. ${ }^{1}$, Cherneta O.G. ${ }^{2}$, Yurov V.M. ${ }^{3}$ \\ 1National University "Zaporizhzhya Polytechnic", Zaporizhia, Ukraine, schmirung@gmail.com \\ 2Dniprovsk State Technical University, Kamenskoye, Ukraine, ocherneta@gmail.com \\ 3E.A. Buketov Karaganda University, Karaganda, Kazakhstan, exciton@list.ru
}

\begin{abstract}
In the work, the regularities of changes in the shear strength of the adhesive bond $\tau$ and the piezoelectric coefficient $\beta$ in the metal systems "steel 45 - 40X", "steel $45+B-40 X "$ ", "steel $45+B C-40 X^{\prime \prime}$ were obtained during physical modeling of the materials I-20A, Wolf 10W-40, TAD-17i, Litol-24 with distinctive dynamic viscosities using the additional equipment of the SMTs-2 friction machine. It has been established that a twofold increase in the shear rate causes a decrease in the piezoelectric coefficient in the "steel 45+VS - 40X" system by a factor of 1.2-1.6 for Wolf 10W-40, TAD-17i, Litol-24 lubricants and its relative similarity independent of dynamic viscosity. At the same time, a twofold increase in the shear rate in the "steel $45+B-40 X^{\prime \prime}$ system also causes a decrease in the piezoelectric coefficient by a factor of 1.16-1.38 for all tested lubricants and its alignments, as in the "steel 45+VS" system, does not observed. It was found that the clearly expressed regularity of the effect of the equivalent dynamic viscosity on the strength of the adhesive bond at $p=0 \mathrm{MPa}$ is not revealed, and the nature of the manifestation is predetermined by a possible change in the mechanism of intermolecular interaction, which depends on the structure of the molecules of lubricants. It was determined that the piezoelectric coefficient naturally decreases with an increase in dynamic viscosity in the entire considered range of normal pressures.
\end{abstract}

Keywords: metal system, tangential strength, shear rate, piezoelectric coefficient, pressure, dynamic viscosity

\section{Introduction}

This work is a continuation of work [1], which shows the importance of having numerical values of the parameters of adhesion properties directly for the surfaces of metals of full-scale operational friction units of machines and mechanisms with reversible movement, ie. in which there is a shift with a certain speed of movement, when the manifestation of the molecular (adhesive) component of the friction force has time to manifest itself. Tribophysical assessment of the state of the near-surface layers of metals of parts working under sliding friction with unidirectional movement is also important. This is due to the fact that when some mechanisms are activated, one surface of the part moves relative to the other. In this case, the force parameter of their contact interaction changes from minimum to maximum and back. Such mechanisms include, for example, the gas distribution mechanism of an internal combustion engine (DVZ), in which the cam displacement relative to the end of the valve or pusher at the initial moment of movement occurs with a normal force due to the magnitude of the compression force of the valve spring when it is opened and closed. The shift rate is predetermined by the starting crankshaft rotation speed and depends on the energy consumption of the DVZ electric start system. In addition, each subsequent shift is carried out under conditions of contact through lubricating formations that were previously formed under conditions of amplitude-frequency change in the force and speed parameters of loading the contact pads of the friction surfaces. The efficiency of the DVZ operation depends on the tribophysical state of the camshaft friction surfaces: power, dynamics, efficiency [2, 3]. In most cases, automotive DVZs use one-piece camshaft structures, which are made of structural mediumcarbon steel with surface wear-resistant layers. Additional hardness of finished shafts is obtained as a result of hardening: nitriding, laser treatment, bleaching, etc. Surface modification is also possible by saturating the surfaces of the camshaft cams with boron and boron carbide, including with subsequent laser treatment, which leads to the production of new near-surface microstructures with predictably improved parameters of friction and wear $[4,5]$. The tribological state of the friction pair "camshaft cam - valve (pusher)" during shear is estimated by the known parameters: shear strength of the adhesive bond $\tau_{0}$ and piezoelectric coefficient $\beta[5$, 9], and the assessment of their manifestation, taking into account the multifactorial influence, seems to be a significant scientific and technical problem in tribology. A particularly significant factor is surface 
modification, for example with boron, boron carbide, of medium carbon steels and its effect on the surface energy, especially of active centers of adsorption of lubricant molecules. And this, in general, determines the nature of the accumulation of fatigue deformations of the contact interaction areas during multiple shears with different intensities of force, speed loading and viscosity of intermediate lubricant formations, and as a result, wear of the cam surface.

\section{Analysis of publications}

The interest in boriding process of specialists from various industries is caused by the unique ratio of mechanical, physical, corrosive and tribological properties of the resulting coatings. During the second half of the 20th century, scientific schools in many countries studied this process. A detailed review of the boriding process of many metals is reflected in the dissertations [6,7]. It is emphasized that over the decades of application of the boriding process, various methods of saturation of products with boron have been proposed, the mechanisms of formation of boride coatings on carbon and alloy steels, their phase composition and properties have been studied. A common disadvantage of all boriding methods is the long duration of diffusion saturation. To solve this problem, methods are being sought to intensify diffusion saturation and reduce costs. The study of adhesive shear in lubricants, which can simulate the gradient viscosity of lubricants with boundary lubrication in friction triads by their viscosity, is a fundamental result, both from a practical and theoretical point of view. There can be three, four or more such lubricants. A lubricant, as an element of a mechanical system, significantly affects the reliability of the entire system, however, during operation, its properties change, therefore, establishing a resource for it is an important problem. The main requirement for lubricants is to ensure the wear resistance of materials of friction pairs in a wide range of loads, speeds and temperatures, therefore, the solution to this problem should be aimed at determining the relationship between the mechanisms of aging of lubricants and wear of materials, the formation of modified protective layers during friction. All these questions are devoted to dissertations [6-8], which contains a list of extensive literature. At the same time, not enough attention has been paid to the issues of assessing the effect of the viscosity of a lubricant on the shear resistance of surfaces when varying the parameters of their modification $[5,9,10]$. This is especially important for materials that have a certain technological heredity of surface properties acquired during the operation of real parts. Such parts include the aforementioned camshaft, made, for example, of 45 mediumcarbon structural steel with an HFC-hardened surface layer. Moreover, such information is not only of scientific and applied interest for the development of tribological and materials science aspects of ensuring the reliability of mechanical engineering objects, but also is the initial data for modeling the patterns of changes in their tribological state and control of processes in them. Simulating changes in the viscosity of lubricant formations in the considered friction pair "camshaft cam - valve (pusher)", the basis of which, as a rule, is a mineral, semi-synthetic, synthetic oil is very difficult due to the complexity of reproducing the viscosity of lubricating formations from the same type of environment.

However, it is possible to simulate using lubricants other than the bases, but with known viscosities. Of course, this approach imposes certain restrictions, but it allows, in the first approximation, to establish the direction of the process. Thus, having the surface structures of the camshaft cam fragments modified by boron, boron carbide and definitely selected lubricants, it seems possible to perform a model assessment of the nature of the change in the parameters of its molecular bond with the valve (pusher) material depending on the viscosity of the interaction medium.

\section{Objective}

The aim of the work is to establish the regularities of changes in the parameters of the adhesive bond in the systems of materials "steel 45-40X", "steel 45+B-40X", "steel 45+BC-40X" in the physical modeling of the shift between small samples in a lubricant environment viscosities using additional equipment of the SMTs-2 friction machine.

\section{Research methodology}

The parameters of the adhesive bond of materials were determined using additional equipment for the SMTs-2 friction machine in accordance with the methodology proposed in [1]. In this case, the force loading parameter was represented by the normal pressing force of the fixed shoe (a fragment of the camshaft cam steel 45) of $140 \mathrm{~N}, 185 \mathrm{~N}, 230 \mathrm{~N}, 280 \mathrm{~N}$. The time of $\mathrm{t}_{1}=0.43 \mathrm{~s}$ and $\mathrm{t}_{2}=1 \mathrm{~s}$ for the angular displacement of the 
shearing disk (material 40X) under the action of the lever mechanism determined the parameter of high-speed loading - the shear rate. The displacement speed of the disk relative to the block was $v_{1}=10.16 \pm 0.8 \mathrm{~mm} / \mathrm{s}, v_{2}$ $=5.08 \pm 0.6 \mathrm{~mm} / \mathrm{s}$. The average linear displacement of the disk was $1=4.5 \mathrm{~mm}$. The chemical composition of the materials of the system under study is presented in Table 1.

Table 1. Chemical composition of steel, $\%$

\begin{tabular}{|c|c|c|c|c|c|c|c|c|c|}
\hline Element & $\mathrm{C}$ & $\mathrm{Si}$ & $\mathrm{Mn}$ & $\mathrm{Ni}$ & $\mathrm{S}$ & $\mathrm{P}$ & $\mathrm{Cr}$ & $\mathrm{Cu}$ & $\mathrm{Fe}$ \\
\hline 45 & $0.42-$ & $0.17-$ & $0.5-0.8$ & before & before & before 0.035 & before & before & $\sim 97$ \\
& 0.5 & 0.37 & & 0.25 & 0.04 & & 0.25 & 0.25 & \\
\hline $40 \mathrm{X}$ & $0.36-$ & $0.17-$ & $0.5-0.8$ & before & before & before 0.035 & $0.8-1.1$ & before & $\sim 97$ \\
& 0.44 & 0.37 & & 0.3 & 0.035 & & & 0.3 & \\
\hline
\end{tabular}

Boring and boron cementing of block samples was carried out in accordance with the methodology described in [4], block samples are shown in Fig. 1. Boronated and boron-cemented surfaces with subsequent laser treatment were not used in the presented studies.

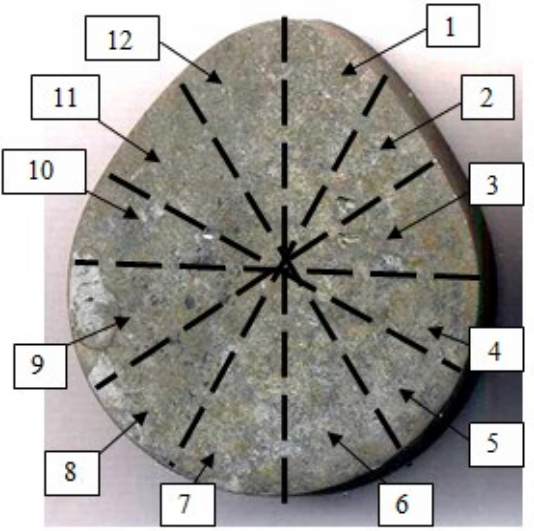

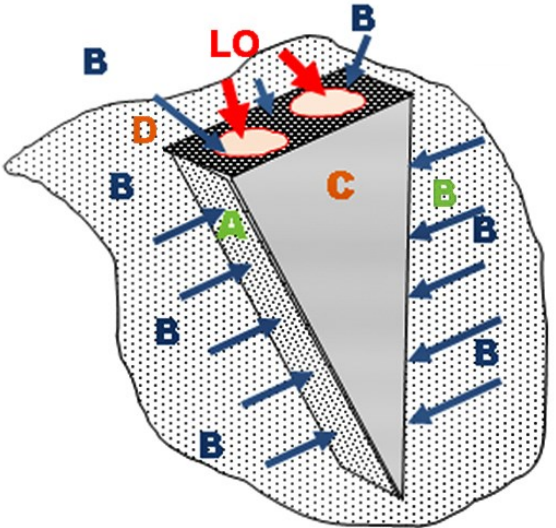

b

Fig. 1. View of samples for experimental research: a - cross-section of the camshaft cam divided into samples-segments No.1-No.12; b - surface treatment scheme: A, B, C, D - plane of the sample-segment; B - boriding (boron cementation); LO - laser processing

The lubricant was applied in a thin layer to the disk at a temperature of $25^{\circ} \mathrm{C}$. In the experiment, we used the following lubricants:

- semi-synthetic motor oil Wolf $10 \mathrm{~W}-40$ API SL/SF, dynamic viscosity at $40{ }^{\circ} \mathrm{C} \mu=0.19 \mathrm{~Pa} \bullet$;

- industrial oil I-20A (GOST 20799-88), dynamic viscosity at $40^{\circ} \mathrm{C} \mu=0.027 \mathrm{~Pa} \bullet$ s;

- transmission oil TAD-17i (GOST 23652-79), dynamic viscosity at $50{ }^{\circ} \mathrm{C} \mu=0.106 \mathrm{~Pa} \cdot \mathrm{s}$;

- Litol-24 grease (GOST $21150-2017$ ), dynamic viscosity at $50{ }^{\circ} \mathrm{C} \mu=8 \mathrm{~Pa} \bullet$ s.

The indicated range of changes in the viscosity of the simulated lubricating medium is quite wide and we think it is significant for experimental studies. At the same time, the lubricants themselves, with the exception of engine oil, cannot be used in the LWD lubrication system. Each of them has its own functional purpose. Motor oil 10W-40 can be used all-season if the temperatures in winter do not drop below $-25^{\circ} \mathrm{C}$, unless the car is equipped with a pre-heater, which eliminates the need to crank the crankshaft with the starter on a cold engine. Industrial oil I-20A is used in units of industrial machines and mechanisms: rolling mills, metal-cutting machines, fans, forging and pressing equipment, textile machines, pumps, etc. The grease is well suited for reducing friction between surfaces with low pressure in light and medium loaded gears, sliding and rolling guides. Oil TAD 17i is a universal all-season transmission fluid that is used to lubricate hypoid, spiral-bevel, worm, cylindrical and bevel gears operating at high loads. The material is suitable for automotive and electric vehicles, transport, trucks and special vehicles. The main advantage of Litola-24 is its high mechanical stability, due to which the material is used in units operating under increased loads. However, if we single out the most significant property for specific conditions and operating modes separately, then they can be equally differentiated taken into account in the contact between the cam and the valve end (valve pusher). Those, we 
can talk about model reproduction of the layer-by-layer viscosity of the forming lubricant formations with a positive gradient to the metal surface. The functionality of the latter is manifested both with boundary and mixed lubrication of friction surfaces. The strength of the adhesive bond at shear (shear resistance of the molecular bond) - tangential strength $\tau$ was determined in accordance with the expression:

$$
\tau=\frac{M_{m}}{r \cdot S_{b}},
$$

where $\mathrm{M}_{\mathrm{m}}$ - moment of friction during shear, $\mathrm{N} \bullet \mathrm{m} ; \mathrm{r}$ is the radius of the disk, $\mathrm{r}=25 \mathrm{~mm} ; \mathrm{Sb}$ - indent area, $\mathrm{Sb}=$ $1.5-5.5 \mathrm{~mm}^{2}$.

\section{Research results and their discussion}

Graphical approximation of the averaged data in the form of linear dependencies with inverse extrapolation of the shear resistance of displacements is shown in Figure 2.
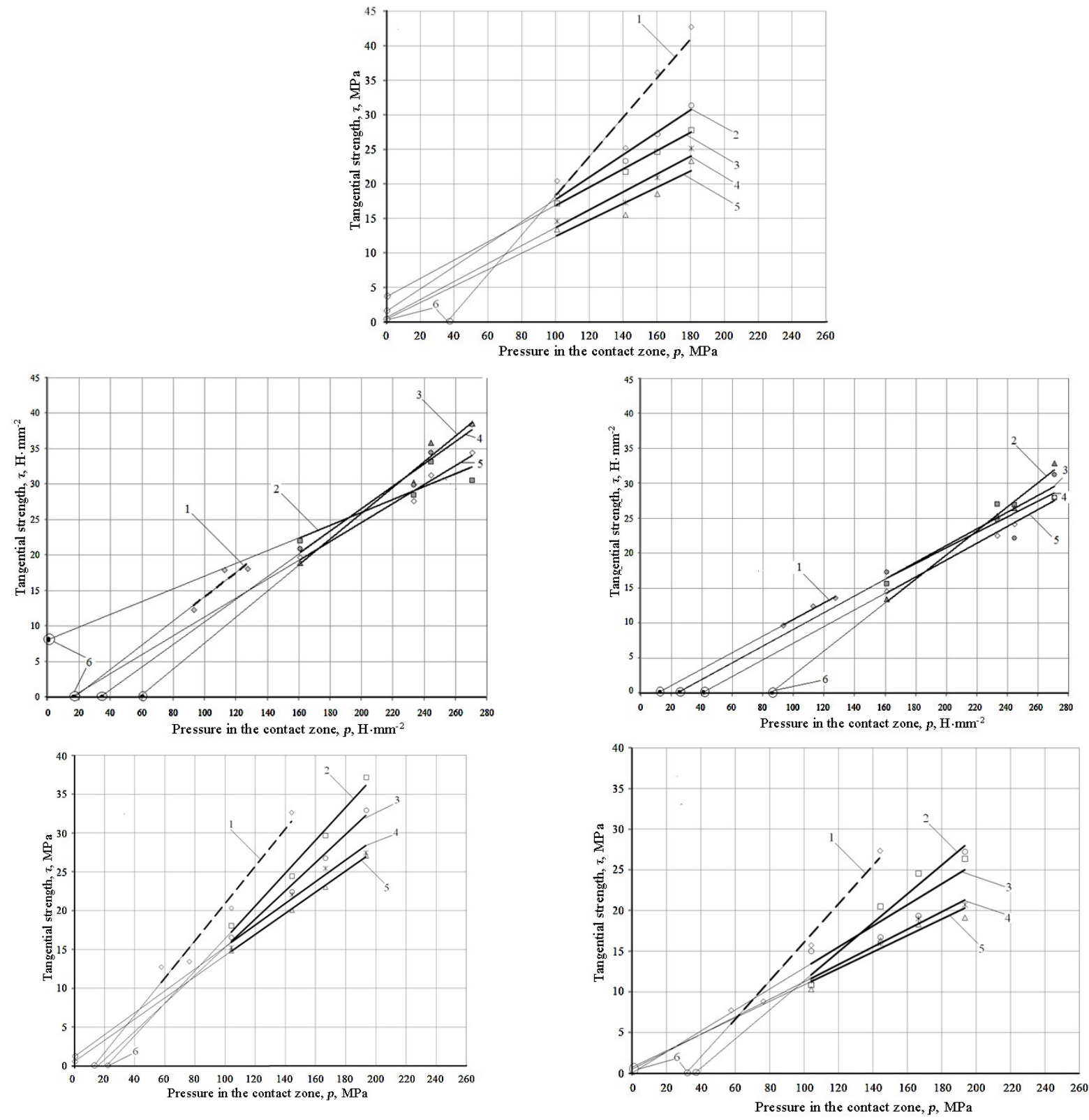

Fig. 2. Tangential strength of the adhesive bond in the contact of materials "40X - steel 45 ": a - steel 45 without treatment; b, c - steel $45+$ BC; d, e - steel $45+$ B; 1 - without lubricant; 2 - I-20A; 3 - Wolf 10W-40; 4 -

TAD-17i; 5 - Litol-24; 6 - extrapolated values of $\tau ; b, d$ - shear rate $5 \mathrm{~mm} / \mathrm{s}$; a, c, d - shear rate $10 \mathrm{~mm} / \mathrm{s}$. 
At the same time, their equations and the accuracy of approximation $\mathrm{R}^{2}$ are determined by the parameters of the trend lines in Excel program, the results are shown in Table 2, 3. Analysis of the data obtained indicates the following. In general, the following picture takes place. The saturation of the surface of steel 45 with boron and boron carbide in almost all cases of shear in lubricating media either leads to a decrease in the values of the piezoelectric coefficient $\beta$, or it remains unchanged: the system "steel $45+$ VS - TAD-17i - 40X" Fig. 2 c, d; system "steel 45 + VS - Litol-24 - 40X" Fig. 2, the exception is the system "steel 45 + VS - I-20A - 40X" at a shear rate $v_{1}=10.16 \pm 0.8 \mathrm{~mm} / \mathrm{s}$, for which the value of $\beta$ increased slightly. A clear decrease in the piezoelectric coefficient $\beta$ is manifested for the unmodified surface of steel 45 during shear in lubricants. Moreover, a smaller value of $\beta$ is typical for Litol 24 with the highest dynamic viscosity, Fig. 2 a. An increase in the shear rate in systems with a modified surface of 45 steel causes its ambiguous influence on the manifestation of adhesion parameters. With regard to the parameter $\tau 0$, there is a tendency to a decrease in its values for systems of materials with modified steel 45 during shear in lubricating media up to the absence of its manifestation at normal pressure $\mathrm{p}_{0}=\mathrm{MPa}$.

Table 2. Parameters of approximation of experimental data for the system of materials "40X - steel $45(+\mathrm{B},+\mathrm{BC})$ " without lubricants

\begin{tabular}{|l|c|c|c|}
\hline \multicolumn{1}{|c|}{ Parameter } & Steel 45 & Steel 45+B & Steel 45+BC \\
\hline \multirow{2}{*}{ Type of equation } & $\tau=0.28 ð-10,27$ & $\tau=0.23 ð-7,5$ & $\tau=0.11$ ठ $-1,23$ \\
\cline { 2 - 4 } & - & $\tau=0.24 ð-3,05$ & $\tau=0.17$ ठ $-3,53$ \\
\hline Approximation reliability $R^{2}$ & 0.9 & 0.96 & 0.97 \\
\cline { 2 - 4 } & - & 0.96 & 0.84 \\
\hline Piezoelectric coefficient $\beta$ & 0.28 & 0.23 & 0.11 \\
\cline { 2 - 4 } & - & 0.24 & $>0$ at $p \approx 11 \mathrm{MPa}$ \\
\hline Tangential strength $\tau_{0}, \mathrm{MPa}$ & $>0$ at $p \approx 36 \mathrm{MPa}$ & $>0$ at $p \approx 32 \mathrm{MPa}$ & $>0$ at $p \approx 21 \mathrm{MPa}$ \\
\cline { 2 - 4 } & - & $>0$ at $p \approx 13 \mathrm{MPa}$ & 0.08 m \\
\hline
\end{tabular}

Note. The upper row of values at a shear rate $v_{1}=10.16 \pm 0.8 \mathrm{~mm} / \mathrm{s}$, the lower one at $v_{2}=5.08 \pm 0.6 \mathrm{~mm} / \mathrm{s}$.

The system "steel $45+\mathrm{VS}-40 \mathrm{X}$ " is characterized by the following manifestation of the adhesive bond parameters, Fig. 2 c, d. An increase in the shear rate by a factor of two causes, firstly, a decrease in the values of the $\beta$ parameter by a factor of 1.2-1.6 for lubricants Wolf 10W-40, TAD-17i, Litol-24, second, its alignments. Those at $v_{1}=10.16 \pm 0.8 \mathrm{~mm} / \mathrm{s}$, the values of the parameter $\beta$ do not depend on the dynamic viscosity. The exception is industrial oil I-20A with the lowest viscosity, for which the value of the parameter $\beta$, on the contrary, increased by 1.8 times. The graph lines on the coordinate field are located in a narrower corridor than the graph lines without modification of steel 45 with boron carbide. This clearly reflects the features of the manifestation of the variable surface energy of the "steel-45 + VS" system on the formation of adhesive bonds with steel 45 through the compacted layers of the lubricant deformed in the interface zone. Such bonds are clearly reduced, and for reliable adhesion it is necessary to increase the pressure in the contact zone, i.e. the tribological system in the region of low contact pressures, for example, less than $20 \mathrm{MPa}$, becomes more reliable.

The system "steel $45+\mathrm{B}-40 \mathrm{X}$ " is characterized by the following manifestation of the adhesive bond parameters, Fig. 2 e, f. An increase in the shear rate by a factor of two causes, firstly, a decrease in the $\beta$ parameter values by 1.16-1.38 times for all tested lubricants, and secondly, its alignments, as in the system "steel $45+\mathrm{BC}$ "is not observed. Those when the shear rate is doubled, there is a proportional decrease in the parameter $\beta$. In this case, the regularity of a decrease in the parameter $\beta$ with an increase in dynamic viscosity remains. The graph lines on the coordinate field are located in a wider corridor relative to the "steel $5+\mathrm{BC}$ " system, and approximately equal to the corridor for placing graphs for unmodified steel 45 .

This clearly reflects the features of the manifestation of the variable surface energy of the "steel- $45+$ B" system on the formation of adhesive bonds with steel 45 through the compacted layers of the lubricant deformed in the interface zone. Such bonds decrease somewhat less than in the "steel $45+$ BC" system. Such a decrease is less pronounced under the action of the components of the TAD-17i and Litol-24 lubricants, i.e. their properties are manifested to provide increased stickiness in the zones of distributed contact. For this system, the range of normal pressures at which the parameter $\tau_{0}>0$ is approximately two times less than in the "steel $45+\mathrm{BC}$ " system. This indicates a positive effect of carbon on a decrease in the strength of the adhesive bond in the considered system of materials. According to table 2, graphical dependences are built, Figure 3, 
which more clearly reflect the nature of the change in the parameters of the adhesive bond in the studied systems of materials.

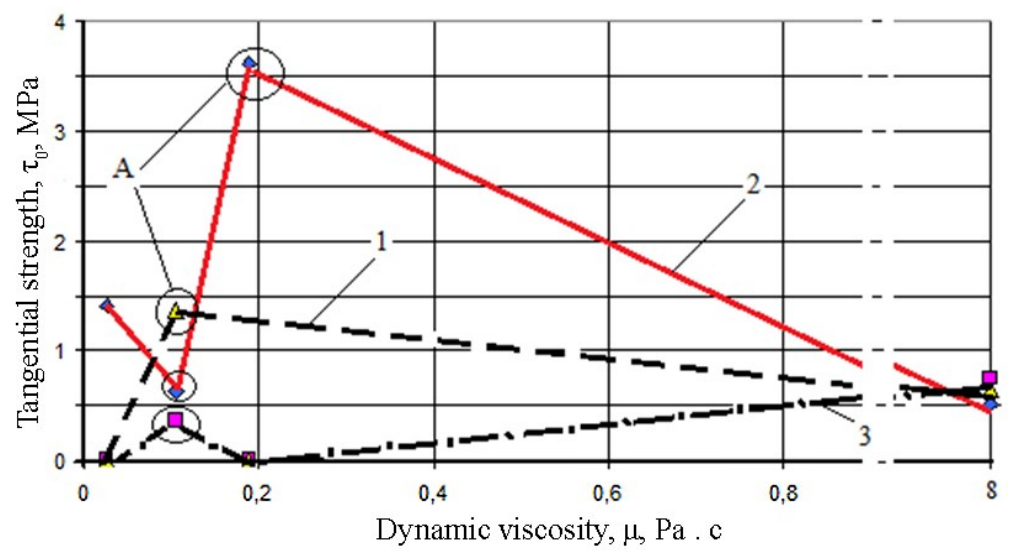

a)

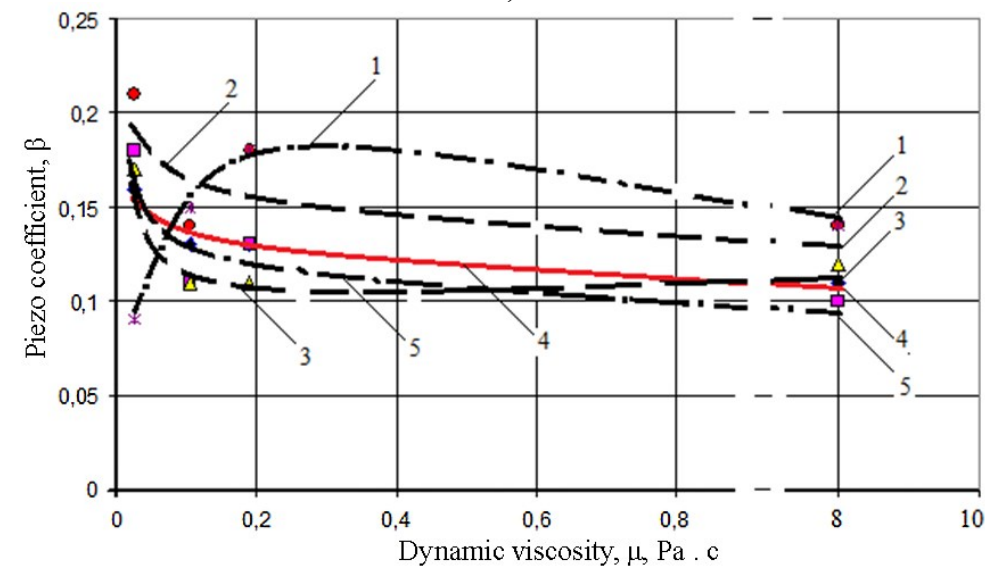

b)

Fig.3. Dependence of the strength of the adhesive bond $\tau_{0}(a)$ and the piezoelectric coefficient $\beta$ (b) on the viscosity of the lubricant: 1 - system "steel $45+\mathrm{BC}-40 \mathrm{X}$ " at $\mathrm{v}_{2}=5.08 \pm 0.6 \mathrm{~mm} / \mathrm{s} ; 2(1 \mathrm{a})$ - "steel $45+\mathrm{B}-40 \mathrm{X}$ " system at $\mathrm{v}_{2}=$ $5.08 \pm 0.6 \mathrm{~mm} / \mathrm{s} ; 3$ - "steel $45+\mathrm{BC}-40 \mathrm{X}$ " system at $v_{1}=10.16 \pm 0.8 \mathrm{~mm} / \mathrm{s} ; 4(2 \mathrm{a})$ - "steel $45-40 \mathrm{X}$ " system at $v_{1}=$

$10.16 \pm 0.8 \mathrm{~mm} / \mathrm{s} ; 5$ (3 a) - "steel $45+\mathrm{B}-40 \mathrm{X}$ " system at $v_{1}=10.16 \pm 0.8 \mathrm{~mm} / \mathrm{s}$; A - change in the mechanism of intermolecular interaction

The analysis of the obtained interpretations of the experimental data indicates the following. No clearly pronounced regularity of the influence of different-grade lubricants with increasing dynamic viscosity on the parameter $\tau_{0}$ was revealed. However, the following trends are visible. First, there is a decrease in the values of the parameter $\tau_{0}$ for unmodified boron steel 45 in two ranges of values of dynamic viscosity: from 0.027 to $0.106 \mathrm{~Pa} \bullet \mathrm{s}$ and from 0.19 to $8 \mathrm{~Pa} \bullet \mathrm{s}$. At the same time, at points A, changes in the mechanisms of manifestation of intermolecular interaction are possible, depending primarily on the structure of the lubricant molecules.

Secondly, there is a decrease in the values of the parameter $\tau_{0}$ for steel modified with boron at a shear rate $v_{2}=5.08 \pm 0.6 \mathrm{~mm} / \mathrm{s}$ in the range of dynamic viscosity values from 0.106 to $8 \mathrm{~Pa} \cdot \mathrm{s}$, and at $v_{1}=10.16 \pm 0.8$ $\mathrm{mm} / \mathrm{s}$ in the range of dynamic viscosity values from 0.106 to $0.19 \mathrm{~Pa} \cdot \mathrm{s}$. In this case, in the range of dynamic viscosity values from 0 to $0.106 \mathrm{~Pa} \mathrm{~s}$, the values of $\tau_{0}$ increase, which also indicates the manifestation of a change in the mechanism of intermolecular interaction, which depends on the structure of the lubricant molecules. Third, for steel 45 modified with boron carbide, the values of the parameter $\tau_{0}$ practically do not appear. An exception is frictional contact in the I-20A lubricant, and even then at low shear rates. For the adhesive bond parameter $\beta$, clearly expressed regularities of a decrease with an increase in dynamic viscosity are established in the entire considered range. The exception is the system "steel $45+\mathrm{BC}-40 \mathrm{X}$ " at $\mathrm{v}_{2}=5.08 \pm$ $0.6 \mathrm{~mm} / \mathrm{s}$ for which the piezoelectric coefficient increases in the range from 0 to $0.19 \mathrm{~Pa} \bullet \mathrm{s}$, and then decreases. This also indicates the manifestation of a change in the mechanism of intermolecular interaction, which depends on the shear rate. In this case, regardless of the nature of the modification of steel 45 (saturation with 
boron or boron carbide), at a higher shear rate, a tendency of a regular decrease in $\beta$ values with respect to its non-modification, and an increase at lower shear rates, appeared.

Table 3. Parameters of approximation of experimental data for the system of materials "40X - steel $45(+\mathrm{B},+$ $\mathrm{BC}) "$ in the environment of lubricants

\begin{tabular}{|c|c|c|c|c|c|c|c|c|c|c|c|c|}
\hline Parameter & \multicolumn{4}{|c|}{ Steel 45} & \multicolumn{4}{|c|}{ Steel 45+B } & \multicolumn{4}{|c|}{ Steel 45+BC } \\
\hline Lubricant & 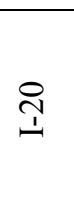 & 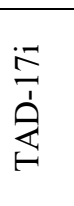 & $\begin{array}{l}9 \\
9 \\
0 \\
0 \\
4 \\
0 \\
3 \\
3\end{array}$ & 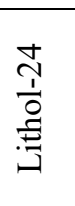 & $\stackrel{\text { I }}{\perp}$ & $\frac{\vec{E}}{\vec{b}}$ & $\begin{array}{l}\frac{9}{9} \\
3 \\
0 \\
4 \\
0 \\
3 \\
3\end{array}$ & 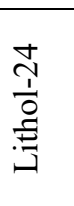 & Iิ & $\frac{i}{E}$ & $\begin{array}{l}9 \\
9 \\
3 \\
0 \\
4 \\
0 \\
3 \\
3\end{array}$ & $\begin{array}{l}\stackrel{ \pm}{ \pm} \\
\text { I } \\
\stackrel{\Xi}{\Xi}\end{array}$ \\
\hline \multirow[t]{2}{*}{ Piezo coefficient $\beta$} & 0.16 & 0.13 & 0.13 & 0.11 & 0.18 & 0.11 & 0.13 & 0.1 & 0.17 & 0.11 & 0.11 & 0.12 \\
\hline & - & - & - & - & 0.21 & 0.14 & 0.18 & 0.14 & 0.09 & 0.15 & 0.18 & 0.14 \\
\hline \multirow[t]{2}{*}{$\begin{array}{l}\text { Tangential strength } \tau_{0} \text {, } \\
\mathrm{MPa}\end{array}$} & 1.4 & 0.63 & 3.61 & 0.51 & $\begin{array}{l}>0 \text { at } \\
p \approx 38 \\
\mathrm{MPa}\end{array}$ & 0.36 & 0.01 & 0.75 & $\begin{array}{l}>0 \text { at } \\
p \approx 86 \\
\mathrm{MPa}\end{array}$ & $\begin{array}{l}>0 \text { at } \\
p \approx 24 \\
\mathrm{MPa}\end{array}$ & $\begin{array}{l}>0 \text { at } \\
p \approx 26 \\
\mathrm{MPa}\end{array}$ & $\begin{array}{l}>0 \text { at } \\
p \approx 42 \\
\mathrm{MPa}\end{array}$ \\
\hline & - & - & - & - & $\begin{array}{l}>0 \text { at } \\
p \approx 20 \\
\mathrm{MPa}\end{array}$ & 1,36 & $\begin{array}{l}>0 \text { at } \\
p \approx 13 \\
\mathrm{MPa}\end{array}$ & 0.64 & 7.88 & $\begin{array}{l}>0 \text { at } \\
p \approx 35 \\
\mathrm{MPa}\end{array}$ & $\begin{array}{l}>0 \text { at } \\
p \approx 40 \\
\mathrm{MPa}\end{array}$ & $\begin{array}{l}>0 \text { at } \\
p \approx 18 \\
\mathrm{MPa}\end{array}$ \\
\hline
\end{tabular}

Note. The upper row of values at a shear rate $v_{1}=10.16 \pm 0.8 \mathrm{~mm} / \mathrm{s}$, the lower one at $v_{2}=5.08 \pm 0.6 \mathrm{~mm} / \mathrm{s}$.

The applied application of the obtained experimental data to the "camshaft cam - valve (pusher)" friction pair indicates the following. The saturation of steel 45 with boron carbide is more significant, since this predetermines a decrease in the strength of the adhesive bond during shear at sufficiently high normal pressures $\mathrm{p}_{\mathrm{i}}>26 \mathrm{MPa}$, and, accordingly, the intensity of the accumulation of fatigue deformations in the near-surface layers. As a result of this contacting process, the wear of the cam material is reduced.

At the same time, the definable values of normal pressures also predetermine the requirements for the operational state of friction surfaces, on which it is necessary to avoid the formation of micro- and submicrosmall contact areas that predetermine the normal pressures of the indicated values, Table 2 . This behavior of the tribological system "40X - steel 45" consists in the features of its surface energy. E. Rabinovich [11] proposed the dependence of the relationship between the friction coefficient on the surface energy of solids. According to Rabinovich, the coefficient of friction $\mathrm{f}$ :

$$
\mathrm{f}=\frac{\mathrm{S}}{\mathrm{p}}\left(1+\frac{2 \mathrm{~W}_{\mathrm{ab}} \tilde{\mathrm{ntg} \theta}}{\mathrm{pr}}+\Lambda\right),
$$

where $\mathrm{S}$ is the shear resistance of the welding bridges; $\mathrm{p}$ is the effective voltage on the contact; $\mathrm{W}_{\mathrm{ab}}$ is the adhesive bond energy; $\theta$ is the angle of inclination of a single irregularity; $r$ is the average radius of the touch spot.

Rabinovich noted that adhesion is especially intense, provided that the bodies have "smooth" surfaces. In this case, the surface energy has a significant effect on the value of the friction coefficient. Taking this into account, Rabinovich points out that the ratio of the adhesion energy and the effective contact stress is an important factor for the selection of friction pairs. If the given expression is large, then the conditions for sliding are bad. In the works of V.D. Kuznetsov, the friction process is considered from the energy side [12]. K. Johnson [13] defined the friction force as a combination of energy and mechanical components

$$
\mathrm{F}=\mathrm{F}_{0}+\sqrt{\frac{8}{\pi}} \mathrm{S}^{3 / 2} \sqrt{\sigma \mathrm{E}},
$$

where $\mathrm{F}_{0}$ is an external force; $\sigma$ is the specific surface energy of the material; $\Delta \mathrm{s}$ is the surface area where the force is applied; E - elastic modulus of the material.

In [14], within the framework of the thermodynamic approach for the dry friction coefficient, we obtained the following formula:

$$
\mathrm{f}=\tilde{\mathrm{N}} \cdot \mathrm{O} \cdot \frac{\sigma \cdot \mathrm{S}}{\Delta \mathrm{G}^{0}} \cdot \overline{\mathrm{N}},
$$


where $\sigma$ is the specific surface energy of the material, $\mathrm{S}$ is the contact area, $\mathrm{T}$ is the temperature, $\Delta \mathrm{G}^{0}$ is the Gibbs energy, $\bar{N}$ is the average number of elementary fracture carriers (proportional to the number of defects), $\mathrm{C}$ is a constant.

All expressions (2) - (4) differ from each other, but with an increase in the surface energy, the friction coefficient (adhesion energy) increases. In table 4 , for steels 45 and $40 \mathrm{X}$, these values are indicated at a temperature close to the melting temperature $\mathrm{Tm}$.

Table 4. Specific surface energy for steels 45 and $40 \mathrm{X}$

\begin{tabular}{|c|c|c|}
\hline Steel & $\mathrm{T}_{\mathrm{m}}, \mathrm{K}$ & $\sigma, \mathrm{J} / \mathrm{m}^{2}$ \\
\hline 45 & 1103 & 0.772 \\
\hline $40 \mathrm{X}$ & 1143 & 0.800 \\
\hline
\end{tabular}

In $[15,16]$, it was emphasized that when analyzing the energy picture of the surface layer, one should proceed from the assumption that approximately $25 \%$ of the total energy is accumulated in the layer. The remaining $75 \%$ of the energy is heat, which comes to the layer and leaves it according to other dependencies. However, the remaining $25 \%$ of the energy creates properties that are undoubtedly among the properties that determine the quality of the product. Roughness in combination with sub-roughness significantly affects the surface energy. The magnitude of the surface energy changes the magnitude and nature of the layer of the adsorbed zone, as well as the zone of oxides, etc. Under its influence, the boundary zone can take different positions relative to the main material of the work piece or part. The energy state of the surface layer to the greatest extent depends on the impact on it in the process of technological manufacture of the part. This effect turns out to be very strong in the course of procurement operations, when the action of deforming forces, heat, chemical process, etc. are combined. In $[15,16]$, as well as from formulas (2) - (4), it follows that the smaller the difference in the surface energies of mating parts made of different materials and under different technological influences, the less will be the coefficient of friction during their mutual movement, and vice versa. This is exactly what Table 4 indicates.

\section{Conclusion}

The results obtained in the work revealed the features of the manifestation of the shear strength of the adhesive bond and its piezoelectric coefficient in the studied metal systems. The established graphic patterns and parameters of their mathematical approximation made it possible to determine the direction of the processes of adhesive interaction of the surface of 45 steel modified with boron and boron carbide with $40 \mathrm{X}$ steel through compacted lubricating formations with distinctive gradients of dynamic viscosity. It has been established that boriding and boron cementation predetermines the expansion of the range of working normal pressures in lubricating media, excluding the manifestation of the adhesion component of friction, which leads to an increase in the reliability of the operation of friction pairs during shear. The data obtained for Wolf $10 \mathrm{~W}-$ 40 engine oil can be used in analytical calculations for predictive assessment of the effect of the adhesion friction component during the start of the DVZ in the drive of the valve groups of its gas distribution mechanism. It follows from the above formulas that the smaller the difference in the surface energies of the mating parts made of different materials and under different technological influences is, the lower the friction coefficient during their mutual movement, and vice versa.

\section{REFERENCES}

1 Kubich V.I., Cherneta O.G., Yurov V.M. Potential difference of metal machine parts methodology for determining the parameters of adhesional properties of materials on the SMC-2 friction machine. Eurasian Physical Technical Journal, 2019, Vol.16, No.2(32), pp. $78-82$.

2 Denisov A.S. Workshop on the technical operation of vehicles. Moscow, 2012, $280 \mathrm{p}$.

3 Rodionov Yu.V., Sevostyanov A.L. Basics of car repair. Penza: PGUAS, 2014, 300 p.

4 Cherneta O.G., Kubich V.I., Voloshchuk R.G., Averyanov V.S., Shmatko D.Z. Strengthening of the Restored Surface Layer of Steel Parts 45 by Laser Boring. International Journal of Engineering \& Technology (UAE). Science Publishing Corporation, RAK Free Trade Zone. Vol 7, No.4.3 (2018), pp. 71 - 75. 
5 Cherneta O.G., Kubich V.I., Shcherbina M.A., Averyanov V.S., Shmatko D.Z. Research of steel 45 strugging influence on the parameters of adhesion communication with chrome, chrome-nickel steel. Mathematical modeling. Kam'yanske. DDTU, 2019, No. 2 (41), pp. 150-158.

6 Bykova T.M. The influence of the chemical composition of steel on the structure and properties of diffusion boride coatings. Dissertation for the degree of candidate of technical sciences, Yekaterinburg, 2016, $164 \mathrm{p}$.

7 Kovalsky B.I. Methodology for monitoring and diagnostics of lubricants as elements of drive systems of multicomponent machines. Dissertation for the degree of Doctor of Technical Sciences, Krasnoyarsk, 2005, $418 \mathrm{p}$.

8 Davidyan L.V. Intensification of diffusion saturation with boron of carbon and alloy steels during microarc heating. Dissertation for the degree of candidate of technical sciences, Rostov-on-Don, 2019, $128 \mathrm{p}$.

9 Kragelsky I.V., Dobychin M.N., Kombalov V.S. Fundamentals of Friction and Wear Calculations. Moscow, Mechanical engineering, 1977, $526 \mathrm{p}$.

10 Semenov V.I., Shuster L.Sh., Chertovskikh S.V., et al. Influence of the complex parameter of plastic frictional contact and material structure on the strength of adhesive bonds. Friction and wear, 2005. Vol. 26, No.1, pp. $74-79$.

11 Rabinowcz E. Surface Energy Approach to Friction and Wear. Product Engineering, 1965, Vol. 36, No 6, pp.95 99.

12 Kuznetsov V.D. Physics of cutting and friction of metals and crystals. Selected Works. Moscow, 1977, $310 \mathrm{p}$.

13 Johnson K. Mechanics of Contact Interaction. Moscow, Mir, 1989, 510 p.

14 Kubich V.I., Guchenko S.A., Yurov V.M., Zavatskaya O.N. On the coefficient of friction of high-entropy sevenatom alloys. 11th International Scientific. conference. "Chaos and structures in nonlinear systems. Theory and experiment", Karaganda, 2019, pp. $114-118$.

15 Musokhranov M.V., Kalmykov V.V., Malyshev E.N., Zenkin N.V. The energy of the surface layer of metals as a tool for influencing the value of the friction coefficient. Fundamental research, 2015, No. 2, pp. $251-254$.

16 Musokhranov M.V., Kalmykov V.V., Logutenkova E.V., Sorokin S.P. Energy state of the surface layer of machine parts. Modern high technology, 2016, No. 5, pp. 276 - 279. 